\title{
Role of Weak Ties in Link Prediction of Complex Networks
}

\author{
Linyuan Lü \\ Department of Physics, University of Fribourg \\ Fribourg 1700, Switzerland \\ babyann519@hotmail.com
}

\author{
Tao Zhou \\ Joint Laboratory of Complex Systems \& \\ Information Physics, University of Science and \\ Technology of China and University of Fribourg \\ zhutou@ustc.edu
}

\begin{abstract}
Plenty of algorithms for link prediction have been proposed and were applied to various real networks. Among these works, the weights of links are rarely taken into account. In this paper, we use local similarity indices to estimate the likelihood of the existence of links in weighted networks, including Common Neighbor, Adamic-Adar Index, Resource Allocation Index, and their weighted versions. In both the unweighted and weighted cases, the resource allocation index performs the best. To our surprise, the weighted indices perform worse, which reminds us of the well-known Weak Tie Theory. Further experimental study shows that the weak ties play a significant role in the link prediction problem, and to emphasize the contribution of weak ties can remarkably enhance the predicting accuracy.
\end{abstract}

\section{Categories and Subject Descriptors}

H.2.8 [Database Management]: Database ApplicationsData mining; H.3.3 [Information Storage and Retrieval]: Information Search and Retrieval-Information Filtering

\section{General Terms}

Algorithms, Experimentation.

\section{Keywords}

Link prediction, Weak ties theory, Complex networks

\section{INTRODUCTION}

Many complex systems can be well described by networks where nodes present individuals or agents, and links denote the relations or interactions between nodes. Recently, the link prediction of complex networks has attracted more and more attention from computer scientists [6] and physicists [3, 17]. Link prediction aims at estimating the likelihood of the existence of a link between two nodes, based on the observed links and the attributes of the nodes. For example, classical

Permission to make digital or hard copies of all or part of this work for personal or classroom use is granted without fee provided that copies are not made or distributed for profit or commercial advantage and that copies bear this notice and the full citation on the first page. To copy otherwise, to republish, to post on servers or to redistribute to lists, requires prior specific permission and/or a fee.

CNIKM'09, November 6, 2009, Hong Kong, China

Copyright 2009 ACM 978-1-60558-807-0/09/11 ...\$10.00. information retrieval can be viewed as predicting missing links between words and documents [18], and the process of recommending items to a user can be considered as a link prediction problem in the user-item bipartite network [22].

The problem of link prediction can be categorized into two classes: One is the prediction of existed yet unknown links for sampling networks, such as food webs, protein-protein interaction networks and metabolic networks; the other is the prediction of links that may exist in the future of evolving networks, like on-line social networks. In addition, the link prediction algorithms can also be used to generate some artificial links to help the further network analysis, such as the classification problem in partially labeled networks $[11,5]$. Some algorithms based on Markov chains [19, 23, 2] and machine learning $[16,20]$ have been proposed recently, and another group of algorithms are based on the definition of node similarity. In this paper, we concentrate on the latter. Node similarity can be defined by using the essential attributes of nodes, namely two nodes are considered to be more similar if they have many common features. However, the essential features of nodes are usually not available, and thus the mainstream of similarity-based link prediction algorithms consider only the observed network structure. Liben-Nowell and Kleinberg [9] systematically compared some structurebased node similarity indices for link prediction problem in co-authorship networks, and Zhou et al. [21, 10] studied nine well-known local similarity indices on six real networks extracted from disparate field, as well as proposed two new local indices.

Up to now, most studies of link prediction do not take weights of links into consideration. Murata et al. [12] proposed three weighted similarity indices, as variants of Common Neighbors, Adamic-Adar and Preferential Attachment indices respectively. They applied these indices to the networks of Question-Answer Bulletin Boards System, and the results show that with the consideration of weights the prediction accuracy can be enhanced. To our surprise, when we apply the weighted indices to co-authorship networks and the US air transportation network, we find that the weighted indices perform even worse than the unweighted ones. Actually, Liben-Nowell and Kleinberg [9] reported the similar observation for weighted Katz index. These unexpected results remind us of the well-known Weak Tie Theory [7]. Further experimental study shows that the weak ties play a significant role in the link prediction problem, and to emphasize the contribution of weak ties can remarkably enhance the predicting accuracy. 
Table 1: Algorithmic accuracy, measured by precision. Each number is obtained by averaging over 100 implementations with independently random divisions of testing set and probe set. The numbers inside the brackets denote the standard derivations. For example, 0.592(48) means the precision is 0.592, and the standard derivation is 0.048 . The abbreviation, $\mathrm{WCN}^{*}, \mathrm{WAA}^{*}$ and WRA*, represents the highest precisions obtained by Eqs. (7-9), respectively. The corresponding optimal values of $\alpha$ are shown in Table 2.

\begin{tabular}{cccccccccc}
\hline & CN & WCN & WCN* & AA & WAA & WAA $^{*}$ & RA & WRA & WRA* \\
\hline USAir & $0.592(48)$ & $0.443(48)$ & $0.617(45)$ & $0.606(49)$ & $0.517(50)$ & $0.639(48)$ & $0.626(39)$ & $0.558(48)$ & $0.633(41)$ \\
\hline NetScience & $0.822(51)$ & $0.202(37)$ & $0.822(51)$ & $0.957(33)$ & $0.681(43)$ & $0.959(22)$ & $0.962(18)$ & $0.978(14)$ & $0.978(14)$ \\
\hline CGScience & $0.625(59)$ & $0.299(45)$ & $0.782(57)$ & $0.780(49)$ & $0.292(51)$ & $0.917(37)$ & $0.963(15)$ & $0.938(17)$ & $0.969(16)$ \\
\hline
\end{tabular}

\section{DATA AND METHOD}

Considering an undirected simple network $G(V, E)$, where $V$ is the set of nodes and $E$ is the set of links. The multiple links and self-connections are not allowed. For each pair of nodes, $x, y \in V$, we assign a score, $s_{x y}$, according to a given similarity measure. Higher score means higher similarity between these two nodes, and vice versa. Since $G$ is undirected, the score is supposed to be symmetry, say $s_{x y}=s_{y x}$. All the nonexistent links are sorted in the decreasing order according to their scores, and the links in the top are most likely to exist. To test the algorithmic accuracy, the observed links, $E$, is randomly divided into two parts: the training set, $E^{T}$, is treated as known information, while the probe set, $E^{P}$, is used for testing and no information therein is allowed to be used for prediction. Clearly, $E=E^{T} \cup E^{P}$ and $E^{T} \cap E^{P}=\varnothing$. In this paper, the training set always contains $90 \%$ of links, and the remaining $10 \%$ of links constitute the probe set. To quantify the prediction accuracy, we use a standard metric called precision, which is defined as the ratio of relevant items selected to the number of items selected. We focus on the top $L$ predicted links (in this paper, we set $L=100$ ), if there are $L_{r}$ relevant links (i.e., the links in the probe set), the precision equals $L_{r} / L$. Clearly, higher precision means higher prediction accuracy.

The empirical data used in this paper include (i) USAir.the US air transportation network, which contains 332 airports and 2126 airlines (see Pajak Datasets). The weight of a link is the frequency of flights between two airports. (ii) NetScience.- the co-authorship network of 1589 scientists who are themselves working on network science [14]. Here, the weight between two scientists is not simply the number of papers they co-authorized. According to [13], if a paper has $n$ coauthors, then the weight of each pair of authors contributed by this paper is $1 /(n-1)$. For two scientists, the final weight of their link is obtained by summing up the weights contributed by all their co-authorized papers. (iii) CGScience.- the co-authorship network in computational geometry till February 2002 (see Pajek Datasets). This network contains 7343 authors and 11898 links. Two authors are linked if they wrote at least a common paper/book. The weight of a link is assigned by directly counting the number of common papers/books.

\section{UNWEIGHTED SIMILARITIES BASED ON LOCAL INFORMATION}

Among many similarity indices, Liben-Nowell and Kleinberg [9] showed that the Common Neighbors (CN) and AdamicAdar (AA) index [1] perform the best, which has been further demonstrated by systematically comparing CN, AA index with seven other well-known local similarity indices [21].
In addition, Zhou et al. [21] proposed a new index named Resource Allocation (RA) index, which can beat both CN and AA index. Therefore, in this paper, we concentrate on $\mathrm{CN}$, AA index and RA index, whose definitions are as following.

(i) CN. In common sense, two nodes, $x$ and $y$, are more likely to form a link in the future if they have many common neighbors. Let $\Gamma(x)$ denote the set of neighbors of node $x$. The simplest measure of the neighborhood overlap is the directed count:

$$
s_{x y}=|\Gamma(x) \cap \Gamma(y)|,
$$

where $|Q|$ is the cardinality of the set $Q$.

(ii) AA index. It refines the simple counting of common neighbors by giving the lower-connected neighbors more weights, as:

$$
s_{x y}=\sum_{z \in \Gamma(x) \cap \Gamma(y)} \frac{1}{\log k(z)},
$$

where $k(z)$ is the degree of node $z$, namely $k(z)=|\Gamma(z)|$.

(iii) RA index. Considering a pair of nodes, $x$ and $y$, which are not directly connected. The node $x$ can send some resource to $y$, with their common neighbors playing the role of transmitters. In the simplest case, we assume that each transmitter has a unit of resource, and will equally distribute to all its neighbors. As a results the amount of resource $y$ received is defined as the similarity between $x$ and $y$, which is:

$$
s_{x y}=\sum_{z \in \Gamma(x) \cap \Gamma(y)} \frac{1}{k(z)} .
$$

Empirical analysis shows that [21] comparing with $\mathrm{CN}$ and AA, RA can enhance the prediction accuracy measured by the area under a receiver operating characteristic curve (AUC) [8], especially for the networks with large average degrees (in such cases, the difference between RA and AA is large). AUC takes into account the whole ranking, while precision only concentrates on the top $L$ predicted links. As shown in Table 1, subject to precision, RA still performs remarkably better than $\mathrm{CN}$ and AA. Here comes a simple but significant result, the RA index outperforms $\mathrm{CN}$ and the AA index, and thus can find its applications in better characterize the proximity of nodes in networks.

\section{WEIGHTED SIMILARITIES}

The similarity indices mentioned in the last section only consider the binary relations among nodes, however, in the real world, links are naturally weighted, which may represent the transportation load between two airports in a airline network or the number of co-authorized papers in a 

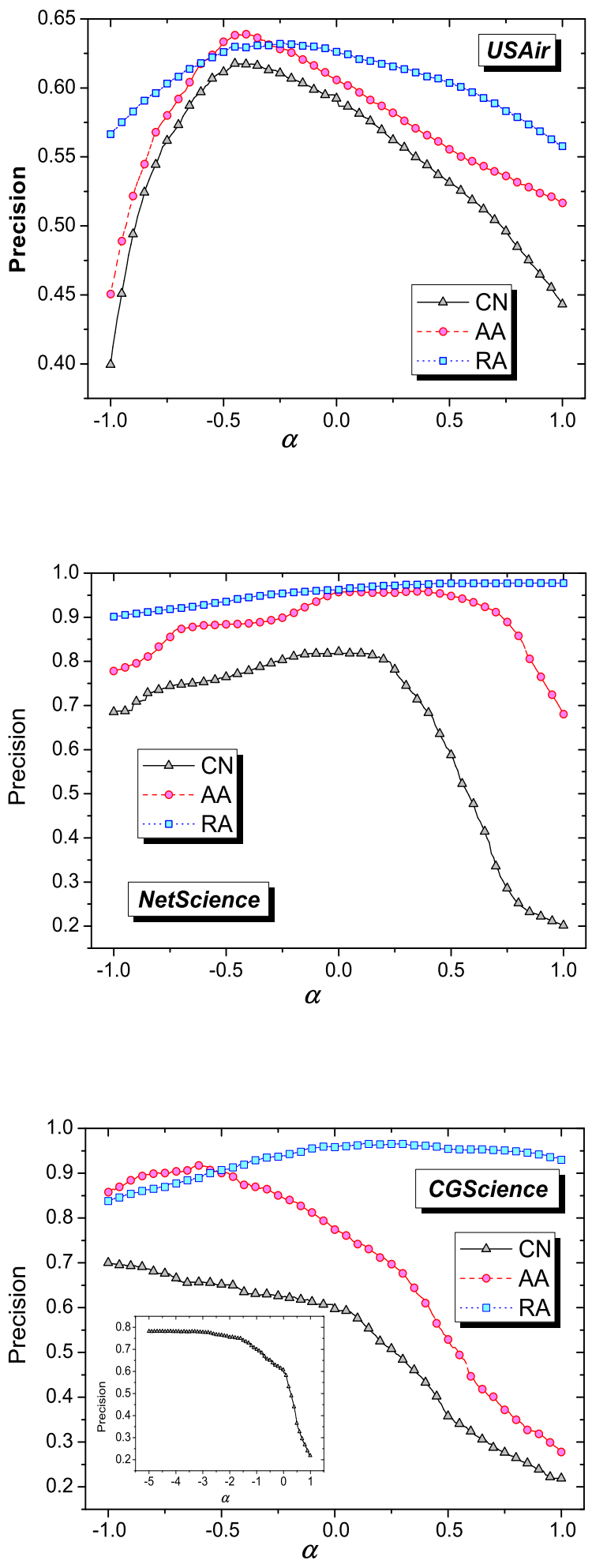

Figure 1: Precision as a function of $\alpha$ for USAir, NetScience and CGScience: CN (triangles), AA (circles) and RA (squares). The inset in the plot for CGScience shows the precision of CN for $\alpha \in[-5,1]$. Each data point is obtained by averaging over 100 realizations, each of which corresponds to an independent division of training set and probe set. co-authorship network. We expect the similarity index taking into account link weights can give better predictions. Murata and Moriyasu [12] proposed a simple way to extend a similarity index for binary network to a weighted index. Following this method, the weighted $\mathrm{CN}$, weighted AA index and weighted RA index (denoted by WCN, WAA and WRA, respectively) are:

$$
\begin{aligned}
& s_{x y}=\sum_{z \in \Gamma(x) \cap \Gamma(y)} w(x, z)+w(z, y), \\
& s_{x y}=\sum_{z \in \Gamma(x) \cap \Gamma(y)} \frac{w(x, z)+w(z, y)}{\log (1+s(z))}, \\
& s_{x y}=\sum_{z \in \Gamma(x) \cap \Gamma(y)} \frac{w(x, z)+w(z, y)}{s(z)} .
\end{aligned}
$$

Here, $w(x, y)=w(y, x)$ denotes the weight of link between nodes $x$ and $y$, and $s(x)=\sum_{z \in \Gamma(x)} w(x, z)$ is the strength of node $x$. Note that, since $s(z)$ may be smaller than 1 we use $\log (1+s(z))$ in Eq. (5) to avoid negative score.

To our surprise, when we apply the weighted indices to the three experimental networks, as shown in Table 1, we find that except WRA in NetScience, the weighted indices perform obviously worse than their corresponding unweighted versions. Especially for $\mathrm{CN}$, with consideration of the weights the precisions are sharply decreased. These unexpected results remind us of the well-known Weak Ties Theory [7], which states that the people usually obtain useful information or opportunities through the acquaintances but not the close friends, namely the weak ties in their friendship network play a significance role. Recently, Onnela et al. [15] demonstrated that the weak ties mainly maintain the connectivity in mobile communication networks, and Csermely found that the weak ties may maintain the stability of biological systems [4]. In contrast, the role of weak ties in link prediction problem has not been investigated yet.

\section{ROLE OF WEAK TIES}

In this section, we provide a start point to investigate the role of weak ties in link prediction by introducing a free parameter, $\alpha$, to control the relative contributions of weak ties to the similarity measure. The parameter-dependent indices for WCN, WAA and WRA are:

$$
\begin{aligned}
& s_{x y}=\sum_{z \in \Gamma(x) \cap \Gamma(y)} w(x, z)^{\alpha}+w(z, y)^{\alpha}, \\
& s_{x y}=\sum_{z \in \Gamma(x) \cap \Gamma(y)} \frac{w(x, z)^{\alpha}+w(z, y)^{\alpha}}{\log (1+s(z))}, \\
& s_{x y}=\sum_{z \in \Gamma(x) \cap \Gamma(y)} \frac{w(x, z)^{\alpha}+w(z, y)^{\alpha}}{s(z)},
\end{aligned}
$$

where $s(x)=\sum_{z \in \Gamma(x)} w(x, z)^{\alpha}$. When $\alpha=0, s(x)$ is the degree of node $x$, and the indices degenerate to the unweighted cases. When $\alpha=1$, the indices is equivalent to the simply weighted indices, as shown in Eqs. (4-6). The numerical results are given in Figure 1, Table 1 and Table 2. For all cases, the optimal values of $\alpha$ are all smaller than 1 . That is to say, the weak links play more important role in the link 
Table 2: Optimal values of the parameter $\alpha$ subject to the highest precisions. For CGScience, with the decreasing of $\alpha$ the precision increases monotonously and eventually reaches a stable value, 0.782 , at the point $\alpha=-4.15$.

\begin{tabular}{cccc}
\hline & WCN $^{*}$ & WAA $^{*}$ & WRA $^{*}$ \\
\hline USAir & -0.41 & -0.40 & -0.24 \\
\hline NetScience & 0.00 & 0.36 & 0.80 \\
\hline CGScience & -4.15 & -0.60 & 0.13 \\
\hline
\end{tabular}

prediction than indicated by their weights. A big surprise is that sometimes the optimal values of $\alpha$ are negative, that is to say, the weak links actually play more important role than the strong links. Although it is well-known that the weak ties mainly maintain the network connectivity, this result is still striking for us.

\section{CONCLUSIONS AND DISCUSSION}

In this paper, we applied three local similarity indices, Common Neighbor, Adamic-Adar index and Resource Allocation index, to the link prediction problem of three empirical networks, USAir, NetScience and CGScience. We found that our previously proposed index, RA [21], performs the best. Furthermore, with the consideration of weights, we tested three weighted variants of $\mathrm{CN}, \mathrm{AA}$ and RA, denoted by WCN, WAA and WRA. To our surprise, the precision of weighted indices perform even worse than their corresponding unweighted versions. These unexpected results remind us the weak ties theory [7] which claims that the links with small weights yet play an important role in social network. Extensive experimental study shows that the weak ties play a significant role in the link prediction problem, and to emphasize the contribution of weak ties can remarkably enhance the predicting accuracy. Sometimes, in the optimal cases, the weak ties contribute more than the strong ties. In another word, the weak links in such network are not as weak as their weights suggested.

Although the prediction accuracies of both the unweighted indices (Eqs. (1-3)) and the simply weighted indices (Eqs. (4-6)) can be further improved by introducing the parameter $\alpha$ (Eqs. (7-9)), this paper does not aim at highlighting these parameter-dependent indices. Instead, we attempt to uncover the role of weak ties in the link prediction problem. We hope this paper can provide a start point for the possible weak ties theory in information retrieval.

\section{REFERENCES}

[1] L. A. Adamic and E. Adar. Friends and neighbors on the web. Social Networks, 25(3):211-230, 2003.

[2] M. Bilgic, G. M. Namata, and L. Getoor. Combining collective classification and link prediction. In Workshop on Mining Graphs and Complex Structures at ICDM-2007, pages 381-386, 2007.

[3] A. Clauset, C. Moore, and M. E. J. Newman. Hierarchical structure and the prediction of missing links in networks. Nature, 453:98-101, 2008.

[4] P. Csermely. Strong links are important - but weak links stabilize them. Trends in Biochem. Sci., 29(7):331-334, 2004.

[5] B. Gallagher, H. Tong, T. Eliassi-Rad, and C. Faloutsos. Using ghost edges for classification in sparsely labeled networks. In SIGKDD'2008, pages 256-264, 2008.

[6] L. Getoor and C. P. Diehl. Link mining: A survey. SIGKDD Explorations, 7(2):3-12, 2005.

[7] M. S. Granovetter. The strength of weak ties. Am. J. Sociology, 78(6):1360-1380, 1973.

[8] J. A. Hanley and B. J. McNeil. The meaning and use of the area under a receiver operating characteristic curve. Radiology, 143(1):29-36, 1982.

[9] D. Liben-Nowell and J. Kleinberg. The link prediction problem for social networks. J. Am. Soc. Inform. Sci. Technol., 58(7):1019-1031, 2007.

[10] L. Lü, C.-H. Jin, and T. Zhou. Effective and efficient similarity index for link prediction of complex networks. arXiv: 0905.3558, 2009.

[11] S. A. Macskassy. Improving learning in network data by combining explicit and mined links. In $A A A I$, pages 590-595, 2007.

[12] T. Murata and S. Moriyasu. Link prediction of social networks based on weightrd proximity measures. $A C M$ International Conference on Web Intelligence, 2007.

[13] M. E. J. Newman. Scientific collaboration networks.ii. shortest paths, weighted networks, and centrality. Phys. Rev. E, 64:016132, 2001.

[14] M. E. J. Newman. Finding community structure in networks using the eigenvectors of matrices. Phys. Rev. E, 74:036104, 2006.

[15] J.-P. Onnela, J. Saramäki, J. Hyvönen, G. Szabó, D. Lazer, K. Kaski, J. Kertész, and A.-L. Barabási. Structure and tie strengths in mobile communication networks. PNAS, 104(18):7332-7336, 2007.

[16] A. Popescul and L. Ungar. Statistical relational learning for link prediction. In Workshop on Learning statistical Models from Relational Data at IJCAI, pages 81-90, 2003.

[17] S. Redner. Teasing out the missing links. Nature, 453:47-48, 2008.

[18] G. Salton and M. J. McGill. Introduction to Model Information Retrieval. MuGraw-Hill, Auckland, 1983.

[19] R. R. Sarukkai. Link prediction and path analysis using markov chains. Computer Networks, 33(1-6):377-386, 2000.

[20] C. Wang, V. Satuluri, and S. Parthasarathy. Local probabilistic models for link prediction. In 7th IEEE International Conference on Data Mining, 2007.

[21] T. Zhou, L. Lü, and Y.-C. Zhang. Predicting missing links via local information. Eur. Phys. J. B, (in press).

[22] T. Zhou, J. Ren, M. Medo, and Y.-C. Zhang. Bipartite network projection and personal recommendation. Phys. Rev. E, 76:046115, 2007.

[23] J. Zhu, J. Hong, and J. G. Hughes. Using markov chains for link prediction in adaptive web sites. LNCS, 2311:55-66, 2002. 